\title{
Avaliação Estatística das Variáveis que Influenciam a Viscosidade da Polpa de Bauxita do Pará com Alto Teor de $\mathrm{SiO}_{2}$ Reativa
}

\author{
Statistical Evaluation of Variables \\ Influencing the Viscosity of the Pulp \\ of Bauxite from Pará with High \\ Content of Reactive $\mathrm{SiO}_{2}$
}

\footnotetext{
${ }^{1}$ Universidade Federal do Rio de Janeiro - Instituto de Química Avenida Athos da Silveira Ramos. 149, CT Bloco A - $7^{\circ}$ andar. Cidade Universitária - Rio de Janeiro - RJ, CEP 21941-909

e-mail: fnogueira@iq.ufrj.br, martam@iq.ufrj.br, chico@iq.ufrj.br

${ }^{2}$ Universidade Federal do Rio de Janeiro -, Escola de Química Avenida Horácio Macedo, 2030, CT Bloco E - sala 200

Cidade Universitária - Rio de Janeiro - RJ, CEP 21941-909

e-mail: cbarbato@peq.coppe.ufrj.br, nele@eq.ufrj.br

${ }^{3}$ Centro de Tecnologia Mineral - Avenida Pedro Calmon, 900 - Cidade Universitária, Rio de Janeiro - RJ - CEP 21941908

e-mail: jsampaio@cetem.gov.br, sfranca@cetem.gov.br
}

\section{RESUMO}

A bauxita do Pará é constituída pelas seguintes camadas: nodular (BN), nodular cristalizada (BNC), cristalizada (BC), cristalizada - amorfa (BCBA) e amorfa (BA). Deste perfil geológico, somente a camada BC é lavrada, beneficiada, transportada através de mineroduto e purificada no processo Bayer. As camadas BCBA e BA possuem um elevado teor de $\mathrm{SiO}_{2}$ reativa (6-7\%), o que inviabiliza sua utilização no processo.

Uma proposta para dobrar as reservas de bauxita, de acordo com o parâmetro comercial, depende de um estudo de caracterização e de beneficiamento que permita identificar as principais dificuldades da utilização da camada BCBA na obtenção de alumina. Assim, este trabalho consistiu na caracterização da bauxita BCBA e no estudo reológico, com o objetivo de verificar a viabilidade desta bauxita para a produção de alumina e do seu transporte por meio de mineroduto.

A caracterização da bauxita BCBA utilizou as técnicas de FRX, DRX, IV, MEV, distribuição do tamanho de partículas por meio de peneiramento, potencial zeta, a análise química pelas técnicas de absorção atômica e titulação potenciométrica. Para estudar a influência da concentração de sólidos, distribuição do tamanho de partículas obtida por diferentes tempos de moagem, temperatura e pH na viscosidade, realizou-se um planejamento de experimento $2^{4-1}$, com ponto central.

A bauxita BCBA é essencialmente gibbsítica e encontra-se associada aos minerais caulinita e hematita. Após as etapas de britagem, moagem e peneiramento, verificou-se que esta bauxita contém um teor de $41,7 \%$ de $\mathrm{Al}_{2} \mathrm{O}_{3}$ disponível e $7,1 \%$ de $\mathrm{SiO}_{2}$ reativa. A DRX e o IV indicaram a caulinita como a fase responsável pelo alto teor de sílica reativa desta bauxita. A avaliação estatística das variáveis de preparo da polpa indicou que a concentração de sólidos é a variável de maior influência na viscosidade, seguida pela influência da distribuição de tamanho de partículas e do $\mathrm{pH}$.

Palavras-chave: Bauxita, Sílica Reativa, Caracterização, Reologia

\section{ABSTRACT}

Bauxite from Pará is constituted by the following layers: nodular (BN), nodular crystallized (BNC), crystal- 
lized (BC), crystallized - amorphous (BCBA) and amorphous (BA). From this geological profile only the BC layer is mined, dressed, transported through pipelines and purified in the Bayer process. The BCBA and BA layers have a high content of reactive $\mathrm{SiO} 2(6-7 \%)$ which makes its use unfeasible to the process.

A proposal to double bauxite reserves, in accordance with the commercial parameter, depends on a study of characterization and beneficiation to identify the main difficulties for BCBA utilization in the Bayer process. Thus, the goals of this work were the characterization of BCBA bauxite as well as the rheological study to verify the feasibility of this bauxite for alumina production and its transport through pipeline.

The characterization of BCBA bauxite used the following techniques: XRF, XRD, IR, SEM/EDS, particle size distribution through wet screening, zeta potential as well as chemical analysis by atomic absorption and potentiometric titration. To study the influence of solids concentration, particle size distribution produced by different grinding times, temperature and $\mathrm{pH}$ on the viscosity of bauxite pulp, a matrix experiment 24-1 around mean values was performed.

The BCBA bauxite is essentially gibbsitic and is associated with kaolinite and hematite. After the steps of crushing, grinding, and screening this bauxite contains $41.7 \%$ of available $\mathrm{Al} 2 \mathrm{O} 3$ and $7.1 \%$ of reactive $\mathrm{SiO} 2$. The XRD and IR identified kaolinite as the phase responsible for the high level of reactive silica in this bauxite. The statistical evaluation of the variables of the pulp preparation indicated that the solid concentration is the most influential variable on viscosity, followed by the influence of particle size and $\mathrm{pH}$.

Keywords: Bauxite, Reactive Silica, Characterization, Rheology.

\section{INTRODUÇÃo}

A bauxita é uma rocha de importância industrial para a obtenção do alumínio metálico. O Brasil, além de possuir grandes reservas (especialmente nos Estados do Pará e de Minas Gerais), é também um dos maiores produtores, ocupando lugar de destaque no cenário mundial. A produção de bauxita é da ordem de 25 milhões de toneladas/ano. Em 2008 os maiores produtores, em ordem decrescente, foram: Austrália, China, Brasil e Índia, com um total de $68 \%$ da produção mundial [1].

A bauxita é formada em regiões tropicais e subtropicais, por ação do intemperismo sobre aluminossilicatos [2]. Apesar de ser frequentemente descrita como o minério de alumínio, a bauxita não é uma espécie mineral propriamente dita, mas um material heterogêneo formado de uma mistura de hidróxidos de alumínio hidratados. Assim, a rocha bauxita compõe-se dos minerais: gibbsita $\left(\gamma-\mathrm{Al}(\mathrm{OH})_{3}\right)$, diásporo $(\gamma-\mathrm{AlO}(\mathrm{OH}))$ e boehmita $(\alpha-\mathrm{AlO}(\mathrm{OH}))$. As proporções desses minerais na rocha variam de acordo com os depósitos. As impurezas contidas na bauxita são os óxidos de ferro: hematita $\left(\alpha \mathrm{Fe}_{2} \mathrm{O}_{3}\right)$, magnetita $\left(\mathrm{Fe}_{3} \mathrm{O}_{4}\right)$ e alumino-goethita $(\alpha(\mathrm{Fe}, \mathrm{Al}) \mathrm{OOH})$, a sílica: caulinita $\left(\mathrm{Al}_{4}\left(\mathrm{SiO}_{4}\right)_{10}(\mathrm{OH})_{8}\right)$ e quartzo $\left(\mathrm{SiO}_{2}\right)$ e os óxidos de titânio: rutilo e anatásio $\left(\mathrm{TiO}_{2}\right)$.

O perfil geológico da bauxita do Nordeste do Pará (Figura 1) é composto de cinco camadas, com diferentes formas de cristalização: nodular (BN), nodular cristalizada (BNC), cristalizada (BC), cristalizada amorfa $(\mathrm{BCBA})$ e amorfa $(\mathrm{BA})$. A bauxita $\mathrm{BN}$ é rica em pisólitos ferruginosos associados à quantidade variável de gibbsita de baixo índice de cristalinidade etambém é composta de hematita, gohetita e caulinita em proporções variadas. Já a bauxita BNC é porosa e contém pisólitos ferruginosos associados à gibbsita de elevado índice de cristalinidade e ricos em hematita. A bauxita $\mathrm{BC}$ caracteriza-se pela predominância de gibbsita com elevado índice de cristalinidade, baixos conteúdos de caulinita e de oxi-hidroxi de ferro e quantidade variável de grãos de quartzo. Na bauxita BCBA o índice de cristalinidade da gibbsita é inferior ao da bauxita BC. Há maior quantidade de materiais argilosos, pouco quartzo e traços de minerais ferrosos. A última camada desse afloramento, bauxita BA, é constituída de materiais argilosos caulíniticos e quantidade variável de grãos de quartzo, além de oxi-hidroxi de ferro. Nesta bauxita há uma quantidade significativa de gibbsita, com índice de cristalinidade elevado.

Deste perfil geológico, somente a camada BC é lavrada, beneficiada, transportada através de mineroduto (244 km de extensão) e purificada por meio do processo Bayer para a produção de alumina. Neste processo, a bauxita é inicialmente moída e, então, dissolvida em meio alcalino $(\mathrm{NaOH})$ sob condições moderadas de temperatura e pressão. Essa reação resulta na formação de uma solução de aluminato de sódio 
$\left(\mathrm{Na}_{2} \mathrm{OAl}_{2} \mathrm{O}_{3}\right)$. Nesta digestão, a sílica reativa, formada por caulinita e quartzo, também é dissolvida e forma o silicato de sódio $\left(\mathrm{Na}_{2} \mathrm{SiO}_{3}\right)$, que reage com a solução de $\mathrm{Na}_{2} \mathrm{OAl}_{2} \mathrm{O}_{3}$ e forma o composto insolúvel sílicoaluminato de sódio $\left(\mathrm{Na}_{2} \mathrm{O} \cdot \mathrm{Al}_{2} \mathrm{O}_{3} \cdot 2 \mathrm{SiO}_{2}\right)$. Este é descartado do processo como lama vermelha ou produto de dessilicação (DSP), o que ocasiona perdas no processo pelo consumo de reagente e produto, $\mathrm{NaOH}$ e $\mathrm{Na}_{2} \mathrm{OAl}_{2} \mathrm{O}_{3}$.

É importante ressaltar que no processo Bayer, há um cuidado especial com a razão $\mathrm{Al}_{2} \mathrm{O}_{3} / \mathrm{SiO}_{2}$, que deve estar próxima de 10 . O alto teor de sílica reativa compromete o processo no contexto da perda de reagente e produto [3]. Já um teor muito baixo de sílica exige a sinterização do minério antes de submetê-lo ao processo, pois sua ausência dificulta a precipitação do DSP [4].

As camadas BCBA e BA são consideradas marginais por possuírem um elevado teor de sílica reativa e outras impurezas, o que inviabiliza a utilização destas camadas no processo Bayer. Uma proposta para dobrar as reservas brasileiras de bauxita, de acordo com o parâmetro comercial, consiste no desenvolvimento de um estudo de caracterização e beneficiamento que torne viável o aproveitamento da camada BCBA para obtenção de alumina.

Neste contexto, este trabalho teve por finalidade a caracterização mineralógica e o estudo do comportamento reológico da camada BCBA, com o objetivo de verificar a viabilidade desta bauxita para a produção de alumina e do seu transporte por meio de mineroduto.

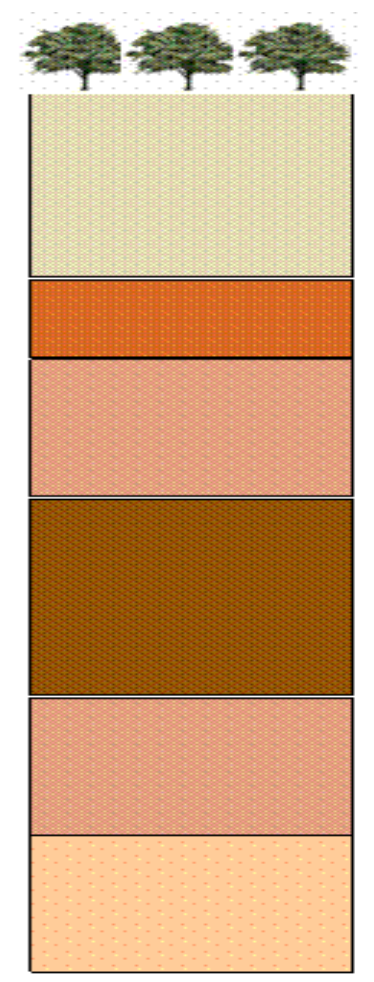

(A)

(B)

(C)

(D)

(E)

Figura 1: Perfil geológico da bauxita do Pará. Em (A) BN, em (B) BNC, em (C) BC, em (D) BCBA e em (E) BA.

\section{MATERIAIS E MÉTODOS}

\section{1 - Preparação da amostra}

A amostra, BCBA, com granulometria inferior a $100 \mathrm{~mm}$, foi homogeneizada em pilha prismática. $\mathrm{Na}$ etapa seguinte, procedeu-se à cominuição da amostra, com o auxílio do britador de mandíbulas. O produto do mesmo foi submetido a uma etapa de peneiramento, na qual se utilizou a peneira de abertura de 1,65 $\mathrm{mm}$. A fração obtida, acima de 1,65 mm, foi rebritada em um britador de rolos, operando em circuito fecha- 
do, com uma peneira de abertura de 1,65 mm. A fração da britagem, inferior a 1,65 mm, foi deslamada para a remoção da fração fina $(-37 \mu \mathrm{m})$. A fração acima de $37 \mu \mathrm{m}$ do processo de deslamagem foi homogeneizada com a fração do britador de rolos, feita uma pilha de homogeneização e foram separadas amostras de $20 \mathrm{~kg}$ (AT). Para realizar os ensaios em laboratório, foi feita nova pilha de homogeneização com uma amostra de $20 \mathrm{~kg}$, obtendo-se alíquotas de $1 \mathrm{~kg}$. Na Figura 2 é apresentado o diagrama de blocos das etapas de beneficiamento utilizadas na preparação da bauxita BCBA.

\section{2 - Análise granulométrica e ensaios de moagem}

Os ensaios de análise granulométrica foram realizados com amostras de $1 \mathrm{~kg}$, segundo o método a úmido [5]. Para tanto, utilizou-se um peneirador vibratório $(684,5 \mathrm{rpm})$, equipado com um conjunto de peneiras de aberturas de $3.350 \mathrm{~mm}$ até $37 \mu \mathrm{m}$, segundo a série Tyler. Todas as frações das análises granulométricas obtidas nos ensaios foram secadas em estufa $\left(100^{\circ} \mathrm{C}\right)$ e pesadas.

Amostras de $1 \mathrm{~kg}$ proveniente do produto final do beneficiamento foram submetidas aos ensaios de moagem, cujo principal objetivo era o de ajustar a amostra às condições necessárias ao processo Bayer. Nos ensaios foi utilizado um moinho de barras de aço inoxidável, contendo dez barras de $20 \mathrm{~mm}$ de diâmetro, com $1 \mathrm{~L}$ de água. Nessa etapa, variou-se o tempo de moagem $(0-30 \mathrm{~min})$. Após cada ensaio de moagem, foram realizados ensaios de análise granulométrica a úmido.

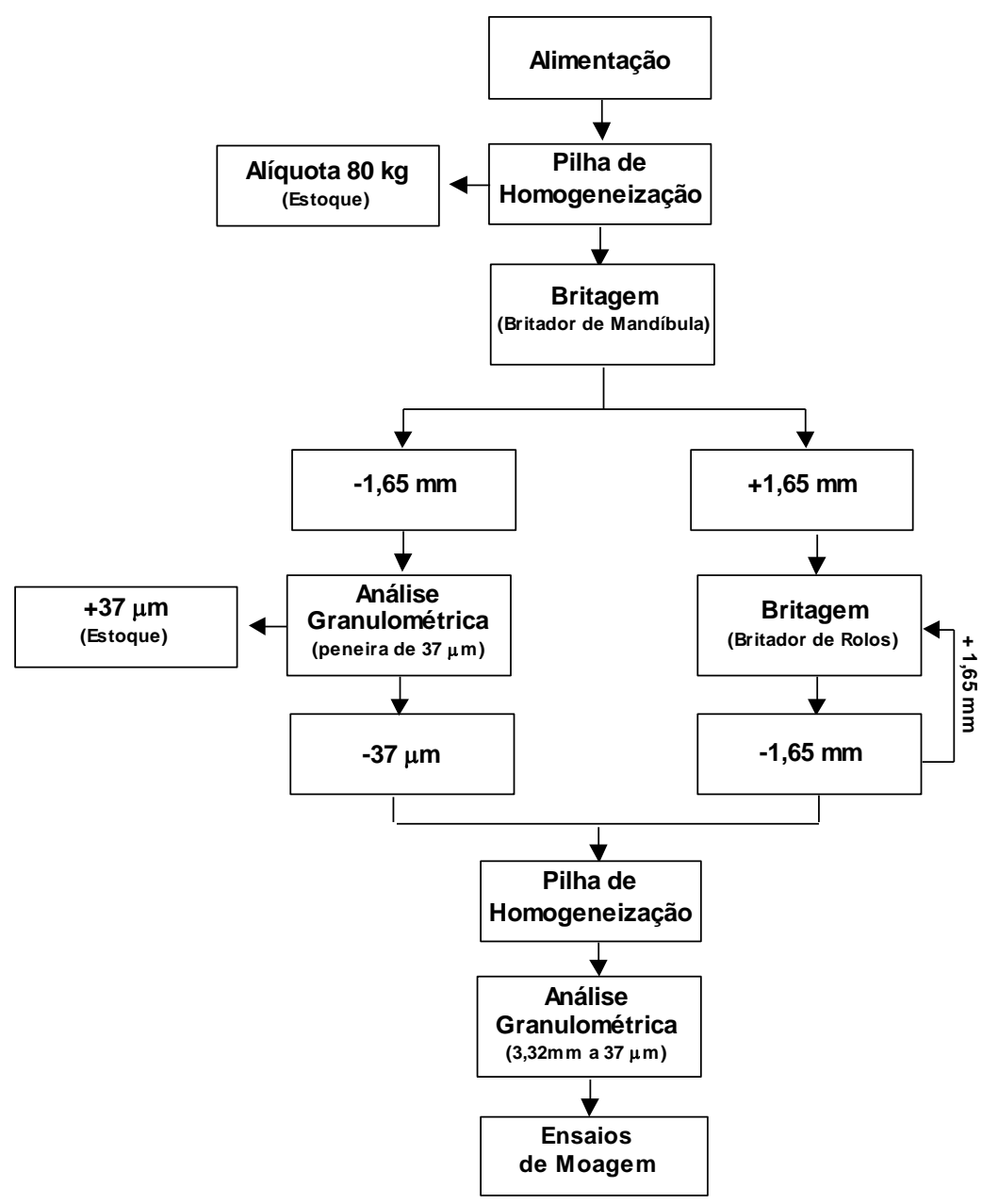

Figura 2: Diagrama de blocos das etapas de beneficiamento utilizadas na preparação da amostra de bauxita BCBA. 


\section{3 - Técnicas de caracterização}

Durante o processo de beneficiamento da bauxita foram geradas as seguintes amostras: bruta de bauxita (Alimentação), a de trabalho (AT), encaminhada para os ensaios de moagem, a da fração $-37 \mu \mathrm{m}$ (Lama) obtida no peneiramento da fração $-1,65 \mathrm{~mm}$ e o produto final do beneficiamento (Moída - M20), obtido após o ensaio de moagem. Outras duas amostras: AT $833 \mu \mathrm{m}$ e AT $208 \mu \mathrm{m}$, foram geradas na análise granulométrica da amostra de trabalho (AT). Por fim, durante os ensaios de moagem, foram geradas três amostras do tipo Moída: M20, M25 e M30, obtidas com o tempo de moagem de 20, 25 e 30 min, respectivamente. Todas as amostras, com exceção das M25 e M30, foram submetidas a uma caracterização química e mineralógica, pelas técnicas descritas a seguir.

\subsection{1 - Análise por Difração de Raios X (DRX)}

Os difratogramas de raios $\mathrm{X}$ das amostras foram obtidos pelo método do pó, em um equipamento Bruker-AXS D5005, com radiação $\mathrm{CoK} \alpha(35 \mathrm{kV} / 40 \mathrm{~mA})$; velocidade do goniômetro de $0,02^{\circ} 2 \theta$ por passo, com tempo de contagem de $1,0 \mathrm{~s}$ por passo e coletados de 5 a $80^{\circ} 2 \theta$.

\subsection{2 - Fluorescência de Raios X (FRX)}

As amostras foram preparadas por fusão do material, a $1.100^{\circ} \mathrm{C}$, utilizando como fundente o tetraborato de sódio na razão 1:6. A pérola fundida foi analisada em espectrômetro de fluorescência de raios $\mathrm{X}$ por energia dispersiva (BRUKER-AXS modelo S4- Explorer), equipado com tubo de Rh. Para obtenção da análise química semiquantitativa, o espectro gerado com base na amostra foi avaliado pelo software Spectra plus v.1.6, no modo standardless method, sem curva de calibração específica.

\subsection{3 - Espectroscopia no Infravermelho (IV)}

A análise por espectroscopia vibracional no infravermelho foi realizada num espectrofotômetro com transformada de Fourier, marca Nicolet Magna, com registros de 4000 a $400 \mathrm{~cm}^{-1}$, resolução de $4 \mathrm{~cm}^{-1}$, em pastilhas de $\mathrm{KBr}$.

\subsection{4 - Determinação da alumina disponível e da sílica reativa}

O método de determinação da alumina aproveitável e da sílica reativa consiste na digestão da bauxita em meio alcalino, sob pressão e temperatura de aproximadamente $8 \mathrm{kgf} / \mathrm{cm}^{2}$ e $149^{\circ} \mathrm{C}$, respectivamente, simulando o processo Bayer [3]. Para a determinação da alumina disponível, adiciona-se ao sobrenadante gluconato de sódio, com o qual se forma um complexo de hidróxido de alumínio - gluconato. Em seguida, é adicionado KF e é realizada uma titulação reversa, isto é, um excesso de solução de $\mathrm{HCl}$ é adicionado e, posteriormente, titulado com solução de $\mathrm{NaOH}$. O resíduo insolúvel, resultante da etapa de digestão do minério que contém a sílica reativa reprecipitada na forma de silicoaluminato de sódio, é dissolvido em solução de $\mathrm{HNO}_{3}$. A determinação de $\mathrm{SiO}_{2}$ reativa foi feita por absorção atômica, no equipamento AA6 Varian, com comprimento de onda de $248,3 \mathrm{~nm}$, fenda de $0,5 \mathrm{~nm}$ e com ar/acetileno.

\subsection{5 - Microscopia Eletrônica de Varredura (MEV/EDS)}

A morfologia dos grãos de bauxita foi determinada em microscópio eletrônico de varredura Leica, modelo F440, em modo de alto vácuo. As amostras foram embutidas em resina epóxi polidas e vaporizadas com carbono para torná-las condutoras e, posteriormente, analisadas por elétrons retroespalhados.

\section{4 - Medidas de Potencial zeta}

O potencial zeta da amostra Moída por 20 min (M20) foi determinado no equipamento PT 1200 fabricado pela Dispersion Technology. As suspensões foram preparadas com 10\% de bauxita e 0,01 $\mathrm{M}$ de $\mathrm{KCl}$. 
BARBATO, C. N.; DA SILVA, F. A. N. G.; SAMPAIO J. A.; MEDEIROS, M. E.;

FRANÇA, S. C. A.; NELE, M.; GARRIDO, F. M. S. revista Matéria, v.18, n.4, pp. 1410-1424, 2013.

As medidas de potencial zeta foram efetuadas nos valores de $\mathrm{pH}$ compreendido entre 2,0 e 12,5. O pH foi ajustado com soluções diluídas de $\mathrm{KOH}$ e $\mathrm{HCl}$.

\section{5 - Determinação da viscosidade das polpas das amostras do tipo moída da bauxita BCBA}

Para avaliar a influência das variáveis de preparo (concentração de sólidos, distribuição de tamanho de partículas obtida por diferentes tempos de moagem, $\mathrm{pH}$ e temperatura) na viscosidade da polpa, realizouse um planejamento de experimentos $2^{4-1}$, com ponto central (Tabela 1). A concentração de sólidos estudada foi igual a 50, 55 e $60 \%$ (p/p), o tempo de moagem da bauxita foi igual a 20, 25 e $30 \mathrm{~min}$, o pH estudado foi igual a 7, 9,5 e 12 e a temperatura foi igual a 25,35 e $45^{\circ} \mathrm{C}$. A viscosidade foi determinada no reômetro ARG2, fabricado pela TA Instruments. Utilizou-se a geometria cilindro coaxial. A programação usada para obter a viscosidade foi a seguinte: duração dos experimentos - $100 \mathrm{~s}$ e taxa de cisalhamento $-200 \mathrm{~s}^{-1}$.

Utilizou-se a Equação 1 para ajustar os valores de viscosidade, tendo como variáveis: a temperatura, o tempo de moagem da bauxita, concentração de sólidos, e pH

$$
Y=a_{0}+\sum_{i}^{4} a_{i} X_{i}+\sum_{i<j}^{4} a_{i j} X_{i} X_{j}
$$

Tabela 1: Condições de preparo das polpas, de acordo com o planejamento de experimentos $2^{4-1}$,com ponto central.

\begin{tabular}{|c|c|c|c|c|}
\hline Ensaios & $\begin{array}{c}\mathrm{T} \\
\left({ }^{\circ} \mathrm{C}\right)\end{array}$ & $\begin{array}{c}\mathrm{TM} \\
(\mathrm{min})\end{array}$ & $\begin{array}{c}\mathrm{CS} \\
(\% \mathrm{p} / \mathrm{p})\end{array}$ & $\mathrm{pH}$ \\
\hline 1 & 25 & 20 & 50 & 7 \\
\hline 2 & 45 & 20 & 60 & 7 \\
\hline 3 & 45 & 30 & 50 & 7 \\
\hline 4 & 25 & 30 & 60 & 12 \\
\hline 5 & 45 & 20 & 50 & 12 \\
\hline 6 & 25 & 20 & 60 & 12 \\
\hline 7 & 25 & 30 & 50 & 9,5 \\
\hline 8 & 45 & 30 & 60 & 9,5 \\
\hline 9 & 35 & 25 & 55 & 9,5 \\
\hline 10 & 35 & 25 & 55 & 9,5 \\
\hline 11 & 35 & 25 & 55 & 9,5 \\
\hline 12 & 35 & 25 & 55 & \\
\hline 13 & 35 & 25 & 55 & \\
\hline
\end{tabular}

Sendo $\mathrm{Y}$ a variável dependente (viscosidade), $\mathrm{X}_{\mathrm{i}}$ são as variáveis independentes (temperatura, tempo de moagem da bauxita, concentração de sólidos e pH) e $\mathrm{a}_{\mathrm{i}}$ e $\mathrm{a}_{\mathrm{ij}}$ são parâmetros.

\section{RESULTADOS E DISCUSSÃO}

A bauxita em estudo, fração BCBA (Figura 1D), foi caracterizada por meio da técnica de difração de raios X (DRX), Figura 3, e as fases mineralógicas que compõem esta rocha foram determinadas. Assim, ela é essencialmente gibbsítica e encontra-se associada aos minerais caulinita $\left(\mathrm{Al}_{4}\left(\mathrm{Si}_{4} \mathrm{O}_{10}\right)(\mathrm{OH})_{8}\right.$ e hematita $\left(\alpha \mathrm{Fe}_{2} \mathrm{O}_{3}\right)$. 
BARBATO, C. N.; DA SILVA, F. A. N. G.; SAMPAIO J. A.; MEDEIROS, M. E.; FRANÇA, S. C. A.; NELE, M.; GARRIDO, F. M. S. revista Matéria, v.18, n.4, pp. 1410-1424, 2013.

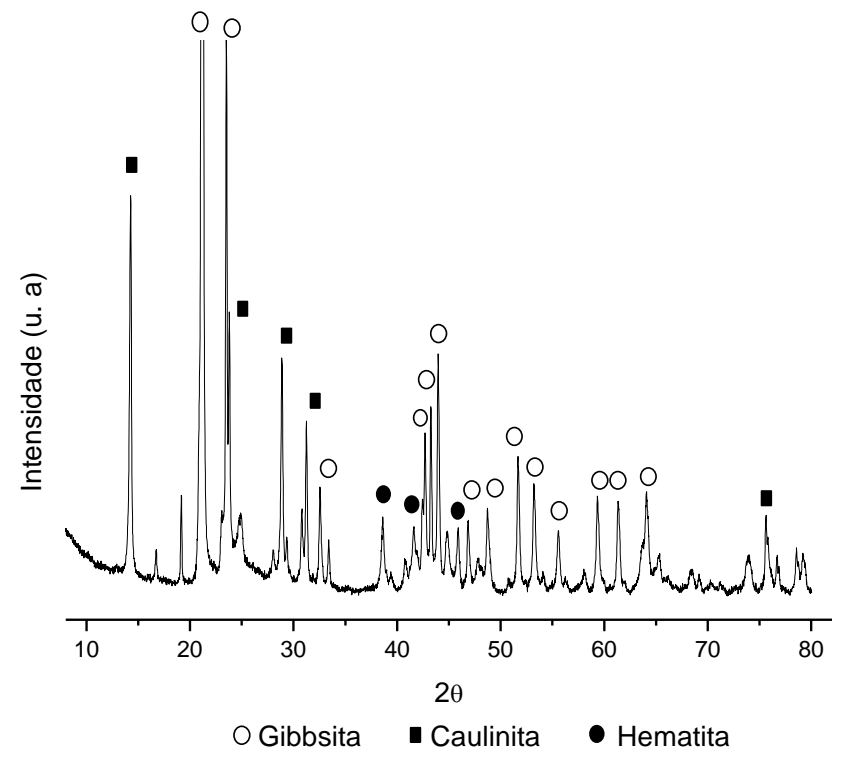

Figura 3: Difratograma de raios $X$ da bauxita BCBA.

A espectroscopia vibracional no infravermelho (IV) foi utilizada como técnica complementar à DRX. Por meio da análise da Figura 4, pode-se observar uma banda em $3.620 \mathrm{~cm}^{-1}$, atribuída ao modo de estiramento Al-O-H, característico da gibbsita e da caulinita $[\underline{3}, \underline{6,10]}$. As bandas observadas em 3.526, 3.459 e $3.394 \mathrm{~cm}^{-1}$ estão relacionadas ao estiramento $\mathrm{O}-\mathrm{H}$ da gibbsita [3]. A banda em $3.696 \mathrm{~cm}^{-1}$ é atribuída ao modo de estiramento $\mathrm{OH}$ da caulinita [9]. Na região entre 1.300 e $400 \mathrm{~cm}^{-1}$, observa-se uma banda em $914 \mathrm{~cm}^{-1}$, atribuída ao modo de deformação do grupo Al-O-H da caulinita [7], e em 968 e $938 \mathrm{~cm}^{-1}$ que são atribuídas à deformação do grupo Al-O-H da gibbsita [10]. Bandas na região de 1.060-1.000 $\mathrm{cm}^{-1}$ estão relacionadas ao modo de deformação do grupo $\mathrm{OH}$ característico da gibbsita $[\underline{8}, \underline{10}]$ e em 538, 667, 748, 796 e $1.116 \mathrm{~cm}^{-1}$ são atribuídas à vibração $\mathrm{Si}-\mathrm{O}[\underline{6}, \underline{7}, \underline{11}]$. As bandas em 470 e $550 \mathrm{~cm}^{-1}$ são características do estiramento $\mathrm{Fe}-\mathrm{O}$ [6].

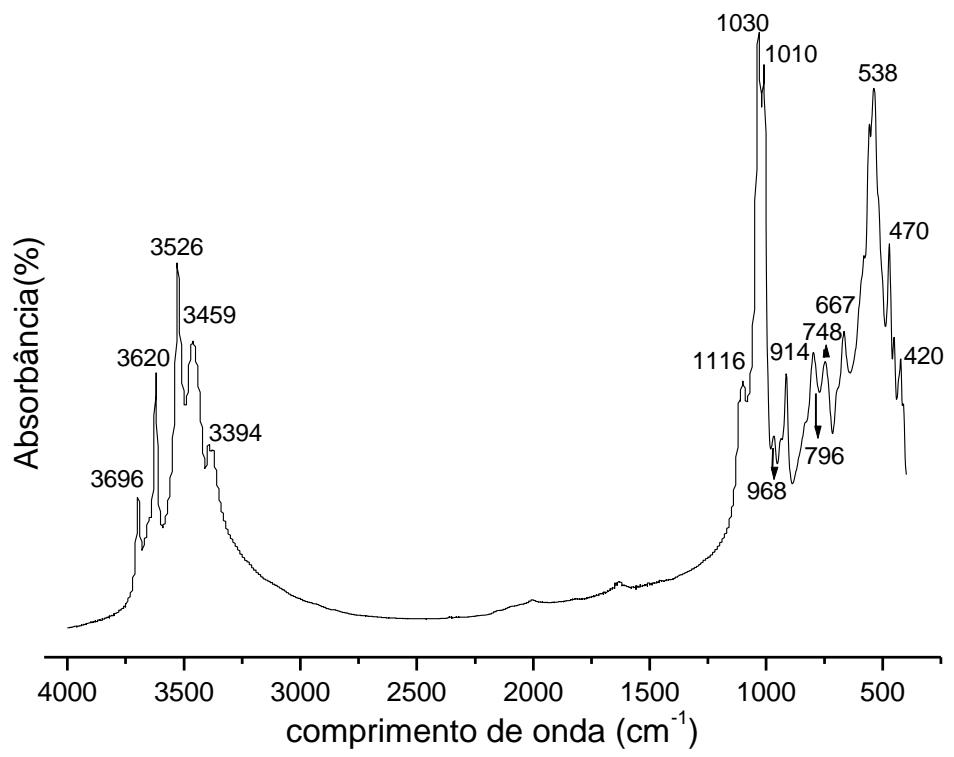

Figura 4: Espectro vibracional na região do infravermelho para a bauxita BCBA. 
BARBATO, C. N.; DA SILVA, F. A. N. G.; SAMPAIO J. A.; MEDEIROS, M. E.;

FRANÇA, S. C. A.; NELE, M.; GARRIDO, F. M. S. revista Matéria, v.18, n.4, pp. 1410-1424, 2013.

Com o objetivo de determinar a composição química da bauxita BCBA e de como os teores variavam de acordo com as etapas de beneficiamento, foi realizada a análise química por FRX, Tabela 2. A análise dos resultados denota que o teor de $\mathrm{SiO}_{2}$ total é maior nas frações mais finas do que nas mais grossas, enquanto um comportamento oposto é observado para o teor de $\mathrm{Al}_{2} \mathrm{O}_{3}$ total. $\mathrm{O}$ teor de ferro encontrado na amostra AT da bauxita BCBA é próximo da utilizada atualmente no processo Bayer (bauxita BC) [12]. Em uma bauxita processada é esperado que o teor de ferro varie entre 5 e $20 \%$, sendo que os minerais ferrosos presentes na bauxita se depositam sobre os grãos de gibbsita e promovem uma coloração ferrugem à rocha $[12, \underline{14}$. Esses minerais também são conhecidos como minerais de ganga, pois durante a dissolução da bauxita gibbsítica permanecem inertes e são removidos da solução com o DSP [4].

Tabela 2: Composição Química por FRX de amostras da bauxita BCBA.

\begin{tabular}{|l|c|c|c|c|c|c|c|c|c|c|}
\hline \multirow{2}{*}{ Amostras } & \multicolumn{10}{|c|}{ Componentes $(\% \mathrm{~m} / \mathrm{m})$} \\
\cline { 2 - 12 } & $\mathrm{Al}_{2} \mathrm{O}_{3}$ & $\mathrm{Fe}_{2} \mathrm{O}_{3}$ & $\mathrm{MnO}$ & $\mathrm{SiO}_{2}$ & $\mathrm{SO}_{3}$ & $\mathrm{TiO}_{2}$ & $\mathrm{WO}_{3}$ & $\mathrm{ZrO}_{2}$ & $\mathrm{PF}$ & Total \\
\hline Alimentação & 46,06 & 9,11 & 0,03 & 14,64 & - & 1,84 & - & 0,22 & 28,10 & 100,00 \\
\hline AT & 53,10 & 8,77 & 0,04 & 9,08 & - & 1,79 & - & 0,25 & 26,97 & 100,00 \\
\hline AT $833 \mu \mathrm{m}$ & 56,54 & 2,25 & - & 5,86 & - & 1,37 & 0,36 & 0,36 & 27,26 & 100,00 \\
\hline AT $208 \mu \mathrm{m}$ & 50,70 & 10,77 & 0,15 & 11,70 & - & 3,92 & - & 0,77 & 21,99 & 100,00 \\
\hline Lama & 40,94 & 12,95 & - & 35,70 & 0,13 & 2,03 & - & 0,28 & 7,97 & 100,00 \\
\hline $\begin{array}{l}\text { AT: amostra obtida após o processo de britagem e homogeneização; } \\
\text { Lama: fração -37 } \mu \text { obtida após o peneiramento da fração -1,65 mm. }\end{array}$
\end{tabular}

De acordo com os resultados de FRX, Tabela 2, observa-se que a Lama, fração -37 $\mu \mathrm{m}$, removida após o peneiramento da fração $-1,65 \mathrm{~mm}$, contém uma quantidade de sílica total igual a 35,70\%. Este alto conteúdo indica que a sílica se concentra preferencialmente nas frações mais finas e é formada essencialmente por caulinita, uma vez que este mineral apresenta granulometria fina, abaixo de $53 \mu \mathrm{m}$. Assim, esta etapa do beneficiamento é essencial ao processo de remoção da sílica reativa.

Com o uso da microscopia eletrônica de varredura por elétrons retroespalhados, Figura 5, foi possível determinar a morfologia dos grãos que compõem a amostra de trabalho (AT). O EDS (Energy Dispersive System) permitiu determinar a composição semiquantitativa desses minerais, Figuras 5A e 5B. Assim, podem ser observados dois tipos distintos de partículas: (A) partículas de gibbsita com superfície lisa e (B) partículas de gibbsita com superfície rugosa, associadas às partículas de caulinita. 


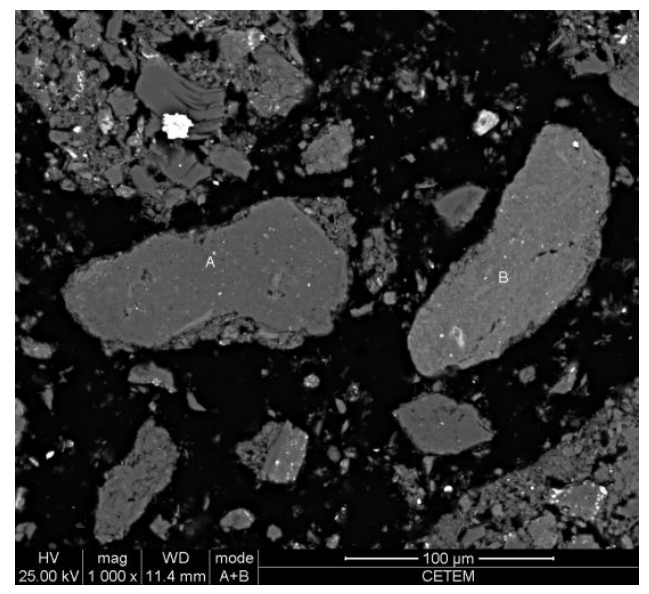

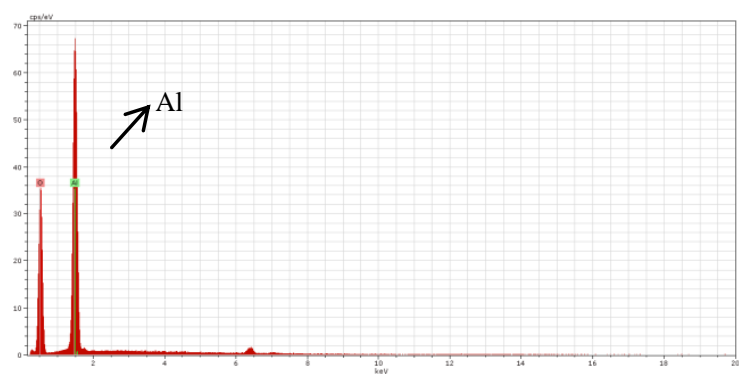

(A)

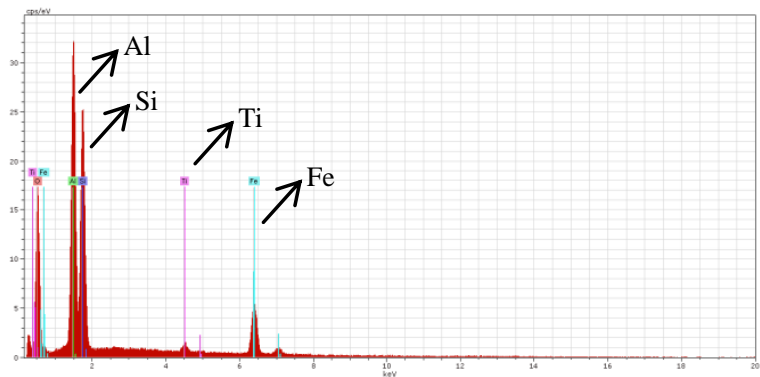

(B)

Figura 5: Imagem da amostra AT, obtida por microscopia eletrônica de varredura por elétrons retroespalhados. Em (A), EDS da gibbsita pura; em (B), EDS da gibbsita associada às partículas de caulinita.

De acordo com os resultados obtidos durante os ensaios de beneficiamento, por meio das técnicas de DRX, IV, FRX e MEV, foi possível constatar a existência de caulinita nas frações mais finas que compõem a amostra bruta (Alimentação) e a amostra AT. Assim, pode-se concluir que a caulinita encontra-se associada às partículas de gibbsita por meio de uma interação de superfície.

Após as etapas de beneficiamento, a amostra AT foi encaminhada para os ensaios de moagem com o objetivo de adequá-la ao processo Bayer, isto é, aproximadamente $90 \%$ das partículas com granulometria inferior a $208 \mu \mathrm{m}$ e $50 \%$ abaixo de $43 \mu \mathrm{m}$. De acordo com a análise dos resultados, Figura 6, o tempo mínimo de moagem é de 20 min para a obtenção da amostra Moída (M).

Segundo as etapas de beneficiamento descritas e o estudo de moagem, a bauxita BCBA apresentou propriedades físicas necessárias à obtenção de alumina pelo processo Bayer. No entanto, para que uma bauxita seja utilizada neste processo é necessária que a mesma apresente uma razão, mássica, de aproximadamente 10:1, entre $\mathrm{Al}_{2} \mathrm{O}_{3 \text { disponível }} / \mathrm{SiO}_{2 \text { reativa. }}$ Para a bauxita em estudo, de acordo com a análise química e utilizando-se um procedimento que simula o processo Bayer [3], a razão da amostra AT foi de 6:1, isto é, 41,7 e 7,1\% de $\mathrm{Al}_{2} \mathrm{O}_{3 \text { disponível }}$ e $\mathrm{SiO}_{2 \text { reativa }}$, respectivamente. Portanto, a quantidade de alumina é menor do que a esperada para o processo, entre 45 e $50 \%$, enquanto que o teor de sílica é maior. A alta quantidade de sílica torna o uso da bauxita da amostra AT inviável ao processo Bayer, pois a sílica consome hidróxido de sódio e aluminato de sódio durante o processo de digestão [4]. Entretanto, os resultados obtidos indicam que, em princípio, é possível ajustar as condições de beneficiamento da bauxita BCBA para que se possa obter uma amostra com um menor conteúdo de sílica reativa, uma vez que para a amostra AT $833 \mu \mathrm{m}$, o conteúdo de sílica total é cerca de metade do conteúdo de sílica total da amostra AT $208 \mu \mathrm{m}$, Tabela 2.resultados similares foram recentemente obtidos para a bauxita BC [12]. 


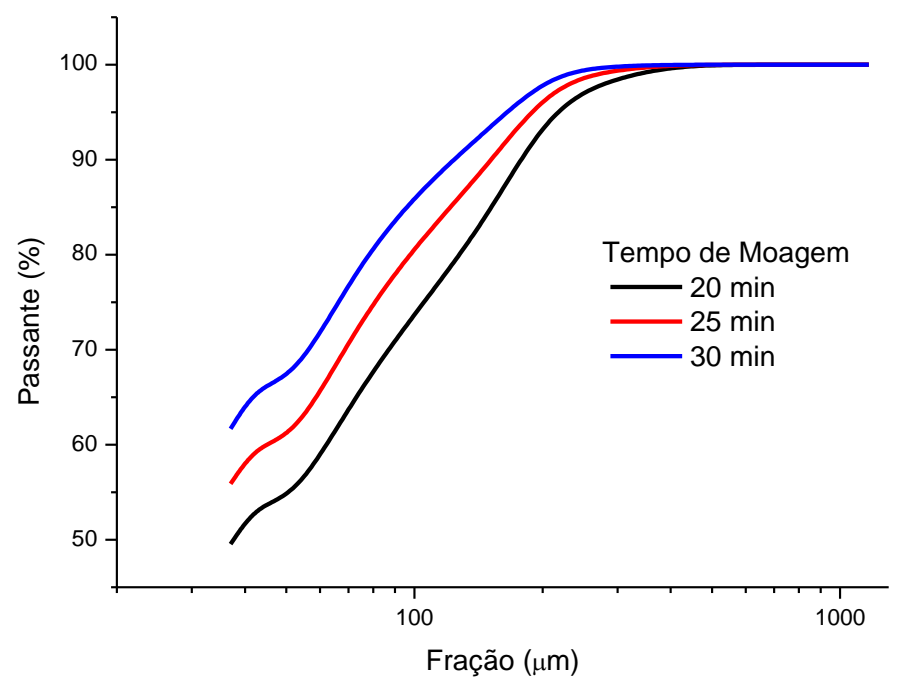

Figura 6: Distribuição granulométrica da bauxita BCBA após 20, 25 e 30 min de moagem.

Para que o transporte de polpas por minerodutos seja economicamente bem sucedido, torna-se necessário que o escoamento da polpa concentrada com maior teor de sólidos possível, seja feito com o mínimo consumo energético. Neste contexto, a descrição do comportamento reológico e a determinação das propriedades reológicas é relevante à otimização das condições do transporte hidráulico da polpa [15]. Uma propriedade reológica importante no processo de bombeamento e transporte de polpas é a viscosidade, um parâmetro importante na definição e escolha das bombas que serão utilizadas. A determinação do perfil da curva de viscosidade deve ser feita dentro de uma faixa de taxas de cisalhamento que inclua as usualmente aplicadas ao processo de bombeamento, situadas em torno de 100 a $200 \mathrm{~s}^{-1}$ [16].

As propriedades reológicas estão relacionadas à estrutura interna da suspensão $[\underline{17}, \underline{20]}$. Assim, para iniciar o escoamento da polpa, é necessária a aplicação de uma força para quebrar os flocos ou agregados formados pela união das partículas, por meio de forças de atração, como por exemplo, a de van der Waals. Neste estudo, verificou-se a influência da concentração de sólidos (50 - 60\%), da distribuição do tamanho de partículas obtida por diferentes tempos de moagem $(20-30 \mathrm{~min})$, da temperatura $\left(25-45^{\circ} \mathrm{C}\right)$ e do $\mathrm{pH}(7-$ 12) na viscosidade da polpa das amostras do tipo Moída da bauxita BCBA. A Figura 7A ilustra os valores da viscosidade da polpa e, também, as curvas de contorno (Figuras 7B e 7C). Verifica-se que a condição experimental que favoreceu o menor valor de viscosidade foi a do ensaio 5 (50\% (p/p) de sólidos, tempo de moagem de 20 min (M20), temperatura de $45^{\circ} \mathrm{C}$ e $\mathrm{pH}$ igual a 12).

Analisando os valores de viscosidade das polpas de bauxita BCBA (Figura 7), pode-se afirmar que há um aumento que varia de acordo com a concentração de sólidos e o tempo de moagem (Figura 7B), e a diminuição da temperatura e do pH (Figura 7C). Este comportamento da viscosidade pode ser explicado pelos itens a seguir:

1. O aumento da concentração de sólidos favorece a diminuição da camada de água entre as partículas. Assim, a distância entre as partículas é menor, o que aumenta a probabilidade de colisões entre elas e, consequentemente, maior quantidade de agregados e empacotamento das partículas $[21,22]$.

2. O aumento do tempo de moagem favorece maior produção de partículas finas $(<37 \mu \mathrm{m})$ (Figura 6). Assim, a bauxita moída por 30 min (M30) contém maior quantidade de partículas finas do que as moídas por 20 e $25 \mathrm{mim}$, cerca de 19,7 e 9,4\%, respectivamente. As partículas finas possuem maior área superficial, o que favorece as interações de van der Walls entre si, inclusive com maior probabilidade de formar aglomerados nos quais as partículas primárias estão unidas e aprisionam água em seu interior. Como esta água não está disponível para o fluxo, a suspensão se comporta como se a fração volumétrica de sólidos fosse maior. 
BARBATO, C. N.; DA SILVA, F. A. N. G.; SAMPAIO J. A.; MEDEIROS, M. E.;

FRANÇA, S. C. A.; NELE, M.; GARRIDO, F. M. S. revista Matéria, v.18, n.4, pp. 1410-1424, 2013.

3. O aumento da temperatura favorece a diminuição da viscosidade da fase dispersante (água), devido ao aumento da mobilidade das moléculas de água.

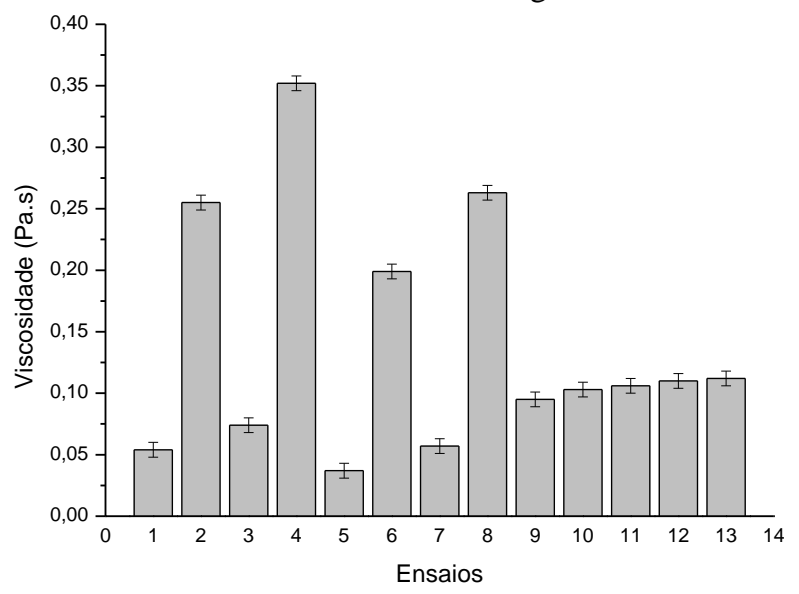

(A)

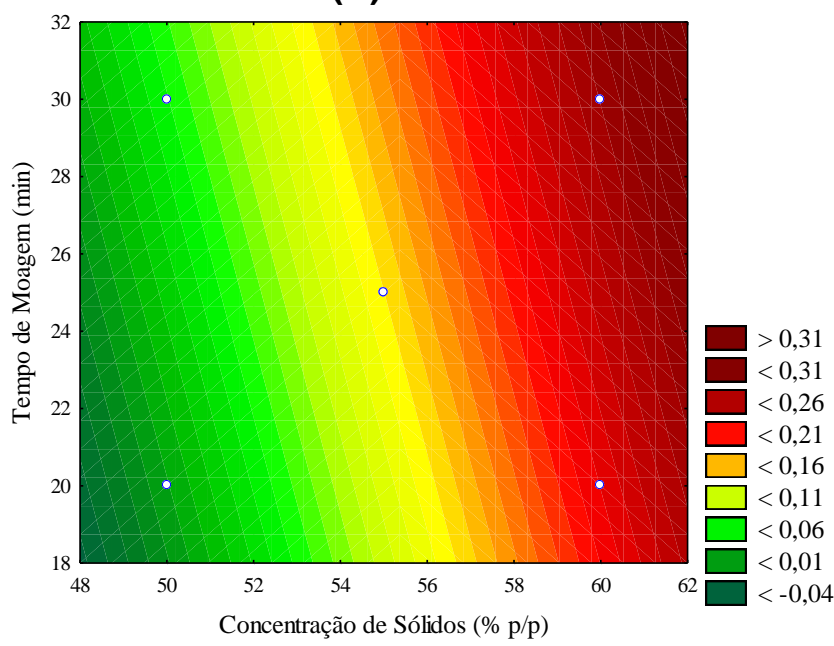

(B)

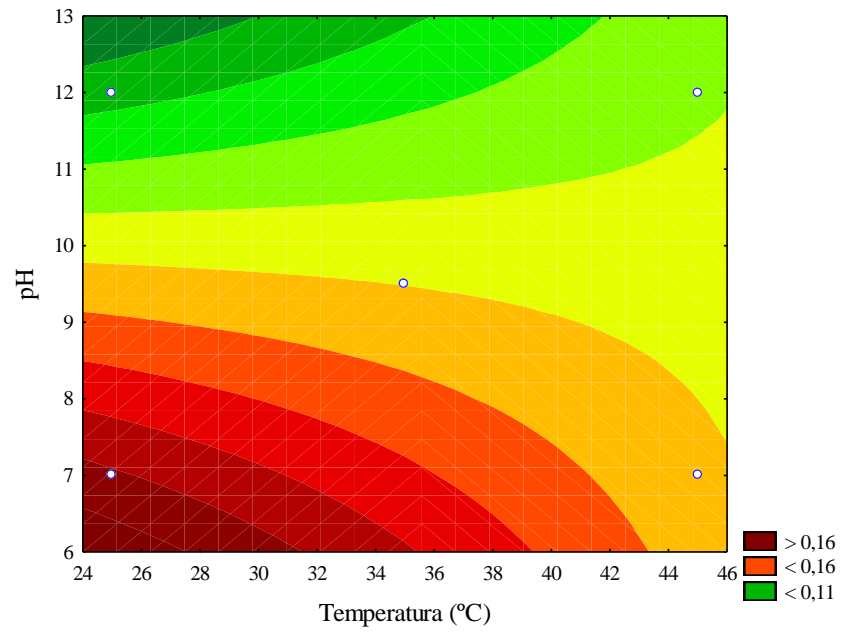

(C)

Figura 7: Em (A), viscosidade das polpas das amostras do tipo Moída, da bauxita BCBA, em (B), curva de contorno na determinação da viscosidade em função da concentração de sólidos e do tempo de moagem, e em (C), curva de contorno na determinação da viscosidade em função do $\mathrm{pH}$ e da temperatura. 
4. O potencial zeta é útil para avaliar as forças elétricas repulsivas e atrativas entre as partículas em uma suspensão[19,23,24]. A Figura 8 ilustra o potencial zeta das partículas da bauxita BCBA em função do pH. No intervalo de pH compreendido entre 7 e 11, o potencial é pequeno. Espera-se que, neste intervalo, o grau de floculação das partículas seja elevado, pois a densidade das cargas na superfície das mesmas é pequena, o que resulta em elevadas forças de atração de van der Waals. No valor de $\mathrm{pH}$ igual ou superior a 12, o potencial zeta é maior, o que significa que a densidade das cargas na superfície das partículas é grande e as forças de repulsão entre elas são maiores que as forças de atração de van der Waals e, consequentemente, um menor grau de floculação. A suspensão terá maior estabilidade e menor viscosidade quando as forças repulsivas são dominantes em relação às forças de atração de van der Waals, responsáveis pela floculação das partículas. Assim, as suspensões preparadas no pH 12 são mais estáveis e menos viscosas do que as preparadas no pH 7 e 9,5, nas mesmas condições de temperatura, distribuição de tamanho de partículas e concentração de sólidos.

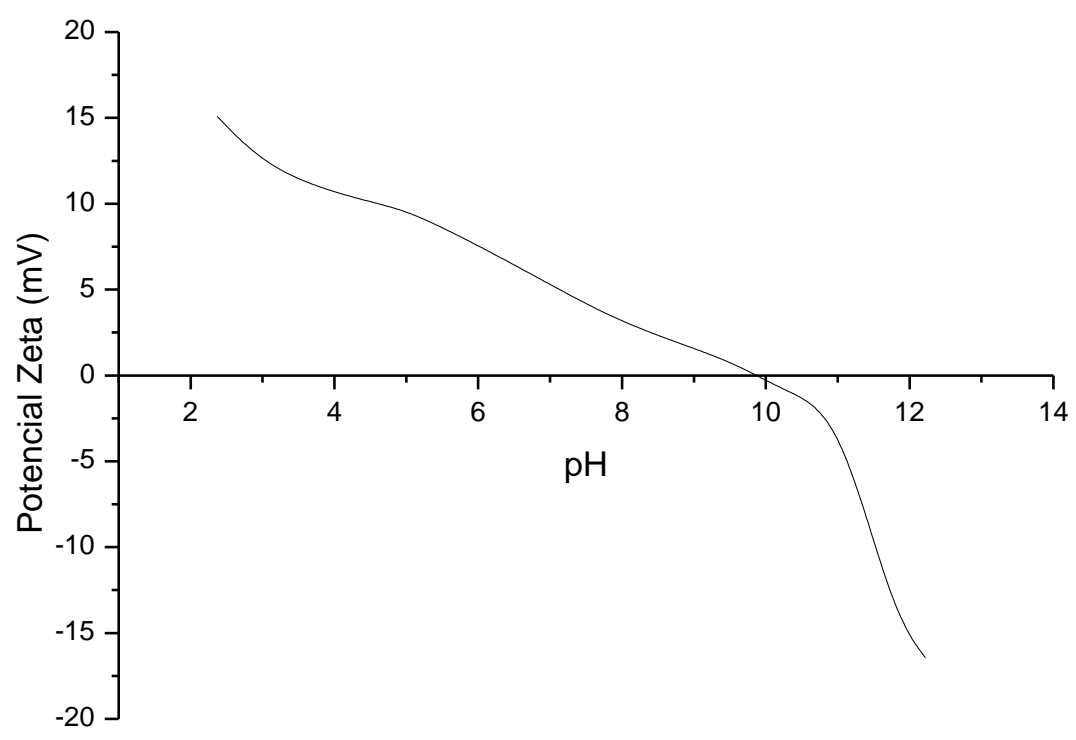

Figura 8: Potencial zeta das partículas da amostra M20 da bauxita BCBA.

A Equação 2 é empírica e relaciona os valores de viscosidade com as seguintes variáveis: concentração de sólidos, tempo de moagem, $\mathrm{pH}$ e temperatura. As variáveis independentes foram normalizadas entre +1 e -1. Desta forma, os valores dos parâmetros podem ser associados ao efeito da variável [25]. A estimação dos parâmetros foi obtida por meio da regressão linear. O teste estatístico padrão de significância (teste $t$ de student) foi utilizado para avaliar a significância do parâmetro. Quando a significância do parâmetro foi menor que 5\%, o parâmetro e seu respectivo efeito foram removidos da Equação 1.

$$
\begin{aligned}
& \eta=0,140 \pm 0,002+0,106 \pm 0,002 \cdot \mathrm{CS}+0,025 \pm 0,002 \cdot \mathrm{TM}-0,022 \pm 0,002 \cdot \mathrm{pH}+ \\
& 0,015 \pm 0,002 \cdot \mathrm{CS} \cdot \mathrm{TM}-0,014 \pm 0,002 \cdot \mathrm{CS} \cdot \mathrm{pH}
\end{aligned}
$$

As análises da Equação 2 e dos dados da Tabela 3 indicam que a concentração de sólidos, o tempo de moagem e o pH influenciam a viscosidade da polpa da amostra que será encaminhada ao processo Bayer da bauxita BCBA. Além disso, verificou-se que o maior valor de viscosidade será obtido quando a concentração de sólidos for igual a $60 \%$, o tempo de moagem igual a $30 \mathrm{~min}$ e o $\mathrm{pH}$ igual a 7 . O efeito da temperatura não foi estatisticamente significante, pois a variação da viscosidade causada pela mudança de temperatura é menor do que o erro experimental. 
BARBATO, C. N.; DA SILVA, F. A. N. G.; SAMPAIO J. A.; MEDEIROS, M. E.;

FRANÇA, S. C. A.; NELE, M.; GARRIDO, F. M. S. revista Matéria, v.18, n.4, pp. 1410-1424, 2013.

A Figura 9 ilustra a comparação entre os valores de viscosidade calculados pela Equação 2 e os obtidos experimentalmente. Observou-se uma ótima aproximação entre os valores de viscosidade calculado e observados. A barra de erro foi calculada com base nos valores de viscosidade de cinco polpas de amostras do tipo Moída da bauxita BCBA, preparadas na condição do ponto central. Verificou-se que a Equação 2 apresenta uma curvatura, pois nas condições experimentais compreendidas entre 1 e 9 os valores observados são maiores que os calculados, enquanto que, no ponto central (condições experimentais de 9 a13), os valores são maiores do que os observados.

Tabela 3: Análise de variância para o planejamento de experimentos 2(4-1)..

\begin{tabular}{|c|c|c|c|c|c|}
\hline Fatores & $\begin{array}{c}\text { Soma dos Qua- } \\
\text { drados }\end{array}$ & $\begin{array}{c}\text { Graus de Li- } \\
\text { berdade }\end{array}$ & $\begin{array}{c}\text { Média Quadrá- } \\
\text { tica }\end{array}$ & F & P \\
\hline $\mathrm{T}$ & 0,001 & 1 & 0,0001 & 3,0 & 0,1559 \\
\hline $\mathrm{TM}$ & 0,0051 & 1 & 0,0051 & 113,0 & 0,0004 \\
\hline $\mathrm{CS}$ & 0,0897 & 1 & 0,0897 & 20006,2 & 0,0000 \\
\hline $\mathrm{pH}$ & 0,0040 & 1 & 0,0040 & 89,6 & 0,0007 \\
\hline $\mathrm{T}^{*} \mathrm{TM}$ & 0,0015 & 1 & 0,0015 & 34,5 & 0,0042 \\
\hline $\mathrm{T}^{*} \mathrm{CS}$ & 0,0001 & 1 & 0,0001 & 3,0 & 0,1559 \\
\hline $\mathrm{T}$ pH & 0,0018 & 1 & 0,0018 & 40,9 & 0,0031 \\
\hline Erro Residual & 0,0097 & 1 & 0,0097 & 40,9 & 0,0031 \\
\hline Erro Puro & 0,0002 & 4 & 0,0000 & & \\
\hline Total & 0,1123 & 12 & & & \\
\hline
\end{tabular}

*Intervalo de Confiança: $95 \%$

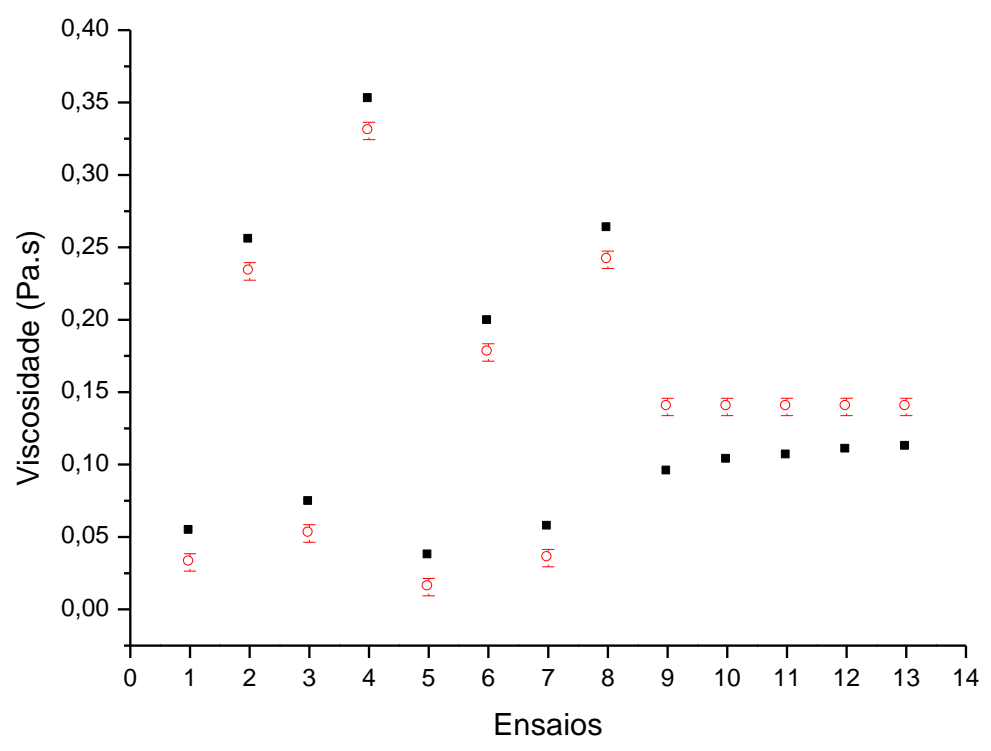

Figura 9- Comparação entre os valores observados ( $)$ e calculados $(\mathrm{O})$ da viscosidade da polpa da amostra Moída da bauxita BCBA.

\section{CONCLUSÕES}

As etapas de beneficiamento tiveram como principal objetivo uma adequação da amostra de bauxita ao processo Bayer. De acordo com os resultados, as etapas de britagem, peneiramento, deslamagem e 20 min. de moagem ajustaram a amostra para o processo Bayer no contexto distribuição granulométrica, isto é, $90 \%$ das partículas com granulometria inferior a $208 \mu \mathrm{m}$ e $50 \%$ menor que $43 \mu \mathrm{m}$.

Os resultados de DRX e IV mostram que a bauxita estudada é essencialmente gibbsítica e está associada aos minerais caulinita e hematita. A FRX forneceu informações relacionadas à composição química da amostra, mas não informou o teor de $\mathrm{Al}_{2} \mathrm{O}_{3}$ disponível e $\mathrm{SiO}_{2}$ reativa, o que tornou necessário a análise quí- 
mica por titulação potenciométrica e absorção atômica. Assim, para esta amostra, os teores de $\mathrm{Al}_{2} \mathrm{O}_{3}$ disponível e $\mathrm{SiO}_{2}$ reativa são 41,7 e $7,1 \%$, respectivamente.

Por meio da microscopia eletrônica de varredura acoplada à análise por EDS foi possível observar dois tipos distintos de partícula: uma de gibbsita pura, superfície lisa, e outra de gibbsita associada às partículas de caulinita, superfície rugosa, o que indica que, mesmo após as etapas de beneficiamento, a caulinita encontra-se associada à gibbsita, possivelmente por meio de uma interação de superfície.

A avaliação estatística das variáveis (concentração de sólidos, tempo de moagem, temperatura e o pH) de preparação da polpa das amostras do tipo Moída da bauxita BCBA, indicou que a variável que tem maior influência na viscosidade é a concentração de sólidos, seguido das influências do tempo de moagem e do $\mathrm{pH}$.

A viscosidade é uma propriedade reológica muito importante, mas não suficiente para definir os parâmetros de bombeamento. Assim, é necessário realizar um estudo mais aprofundado para verificar a influência das variáveis de preparo da polpa em outras propriedades reológicas, tais como a tensão de escoamento, a tixotropia e os módulos elástico e viscoso.

\section{AGRADECIMENTOS}

Os autores agradecem a CAPES e ao CNPq pelo apoio financeiro.

\section{BIBLIOGRAFIA}

[1] BRAY, E. L., U. S. GEOLOGICAL SURVEY, Mineral Commodity Summaries, http://minerals.usgs.gov/minerals/pubs/mcs/2009/mcs2009.pdf , acessado em setembro de 2010.

[2] RIBEIRO, A. G., FALCÃO, A. A, , "O Processamento da Bauxita para a Produção de Alumínio", In: Andrade, J.C., Abreu, M.F. (Eds.) Análises Químicas de Resíduos Sólidos para Monitoramento e Estudos Agroambientais, 1 ed. Cap. 4, p. 47-56, Campinas, São Paulo, Brasil, UNICAMP, 2008.

[3] SILVA, F.A.N.G., MEDEIROS, M. E., SAMPAIO, J. A., et al., "Technological Characterization of Bauxite from Pará-Brazil", In: Bearne, G. (Ed.), Light Metals, Cap. Alumina and Bauxite, p. 139-144, Minerals, Metal \& Materials Society, Pennsylvania, USA, 2009.

[4] SAMPAIO, J.A., ANDRADE, M.C., DUTRA A.J.B., "Bauxita", In: Luz, A.B. da., Lins, F.F (Eds.) Rochas e Minerais Industriais, 2 ed. Cap. 14, Rio de Janeiro, Brasil, Centro de Tecnologia Mineral, 2009.

[5] SAMPAIO, J.A., SILVA, F.A.N.G. “Análise Granulométrica por Peneiramento”. In: Sampaio, J.A., França, S.C., Braga, P.F.A. (Eds.) Tratamento de Minérios Práticas Laboratoriais, Cap. 3, Rio de Janeiro, Brasil, Centro de Tecnologia Mineral, 2007.

[6] MAREL, H.W., BEUTELSPACHER, H., Atlas of Infrared Spectroscopy of Clay Minerals and their Admixture, $1^{a}$ ed, Amsterdam, Elsevier Scientific Publishing Company, 1976.

[7] MENDELOVICI, E. "Selective mechanochemical reactions on dry grinding structurally different silicates". Journal of Materials Science Letters, v. 20, n. 1, p. 81-83, 2001.

[8] SHUMSKAYA, L.G. "Directional Changes in the Properties of Aluminum Hydroxide-Oxides for Increase in Bauxite Reactivity in Hidrometallurgical Processing". Journal of Mining Science, v. 38, n. 3, p.299304, 2002.

[9] SILVA, F.A.N.G. Estudo de Caracterização e Beneficiamento do Caulim da Região Borborema-Seridó $(R N)$, Dissertação de M.Sc., PEMM/COPPE, Universidade Federal do Rio de Janeiro, Rio de Janeiro, 2007.

[10] KLOPROGGE, J.T., RUAN, H.D., FROST, R.L., "Thermal decomposition of bauxite minerals: infrared emission spectroscopy of gibbsite, boehmite and diaspore", Journal of Materials Science, v. 37, n. 6, p. 1121-1129, 2002. 
BARBATO, C. N.; DA SILVA, F. A. N. G.; SAMPAIO J. A.; MEDEIROS, M. E.;

FRANÇA, S. C. A.; NELE, M.; GARRIDO, F. M. S. revista Matéria, v.18, n.4, pp. 1410-1424, 2013.

[11] AZEVEDO, C.A., GARRIDO, F.M.S., MEDEIROS, M.E., "The effect of mechanochemical activation on the reactivity in the $\mathrm{MgO}-\mathrm{Al}_{2} \mathrm{O}_{3}-\mathrm{SiO}_{2}$ system", Journal of Thermal Analysis and Calorimetry, v. 83, n. 3, p. 649-655, 2006.

[12] SILVA, F.A.N.G., SANTOS, R.D., SAMPAIO, J.A., et al., "Study on Ore Dressing and Characterization of Different Granulometric Fractions that Compound Bauxite from Pará/Brazil”, In: Suarez, C., Phillips, E. (Eds.), Light Metals, Cap. Alumina and Bauxite, Minerals, Metal \& Materials Society, Pennsylvania, USA, p. 69-74, 2010.

[13] AQUINO T. F., FÁVERI, R. F, RIELLA, et al., "Caracterização Físico-Química e Mineralógica da Bauxita de Santa Catarina para a Produção de Refratários com Baixo Teor de Óxido Ferro". In: CBECIMat Congresso Brasileiro de Engenharia e Ciência dos Materiais, 17, Foz do Iguaçu, PR, Brasil, 15 a 19 de Novembro de 2006.

[14] KAHN, H., TASSINARI, M.M.L., RATTI, G. "Characterization of bauxite fines aiming to minimize their iron content”. Technical Note, Minerals Engineering, v. 16, n. 11, p.1313-1315, 2003.

[15] NASCIMENTO, C. R., SAMPAIO, J. A. "Propriedades reologicas da polpa de bauxita gibbsitica". In: Encontro Nacional de Tratamento de Minerios e Metalurgia Extrativa, XXIII., Gramado, RS., p. 61-66, 2009.

[16] SAMPAIO, L. C., NASCIMENTO, C. R. "Propriedades reológicas da polpa de bauxita-I Estudo da influência da concentração e distribuição granulométrica". In: Jornada de Iniciação Cientifica-CETEM, XIV. Rio de Janeiro, 2006.

[17] NDLOVU, B., BECKER, M., FORBES, E.,et al. "The influence of phyllosilicate mineralogy on the rheology of mineral slurries”, Minerals Engineering, v. 24, n.12, p. 1314-1322, 2011.

[18] NDLOVU, B, FARROKHPAY, S., BRADSHAW, D., "The effect of phyllosilicate minerals on mineral processing industry”, International Journal of Mineral Processing, v. 125, 149-156, 2013.

[19] YAN, L., MASLIYAH, J. H., XU, Z., "Understanding suspension rheology of anisotropically-charged platy minerals from direct interaction force measurement using AFM", Current Opinion in Colloid \& Interface Science, v. 18,n.2, p. 149-156, 2013.

[20] FARROKHPAY, S., "The importance of rheology in mineral flotation: A review", Minerals Engineering, v. 36-38, p. 272-278, 2012.

[21] NUNTIYA, A., PRASANPHAN, S., "The Rheological behavior of kaolin suspensions”, Chiang Mai Journal of Sience, v. 33, n.3, p. 271-281, 2006.

[22] HE, M., WANG, Y. E FORSSBERG, E., "Slurry Rheology in Wet Ultrafine Grinding of Industrial minerals: a Review", Powder Technology, v. 147, n.1-3, p. 94-112, 2004.

[23] Burdukova, E., Becker, M., Bradshaw, D. J., Laskowski, J. S., "Presence of negative charge on the basal planes of New York talc”, Journal of Colloid and Interface Science, v. 315, n.1, p. 337-342, 2007.

[24] HOU, T., XU, R., ZHAO, A. "Interaction between electric double layers of kaolinite and Fe/Al oxides in suspensions", Colloids and Surfaces A: Physicochemical and Engineering Aspects, v. 297, n. 1-3, p. 9194, 2007.

[25] BOX, G. E. P., HUNTER, W. G., HUNTER, J. S., Statistics for Experiments ,New York, NY: Wiley, 1978 . 
BARBATO, C. N.; DA SILVA, F. A. N. G.; SAMPAIO J. A.; MEDEIROS, M. E.;

FRANÇA, S. C. A.; NELE, M.; GARRIDO, F. M. S. revista Matéria, v.18, n.4, pp. 1410-1424, 2013. 